\title{
Relaciones entre currículo y didáctica: conceptualizaciones, desafíos y conflictos
}

\author{
Relationships between curriculum and didactics. conceptualizations, \\ challenges and conflicts
}

\section{Relações entre currículo e didática: conceptualizações, desafios e conflitos}

\author{
Ángel Díaz-Barriga' \\ Universidad Nacional Autónoma de México, Instituto de Investigaciones sobre la Universidad \\ y la Educación, Investigador Emérito. \\ https://orcid.org/0000-0003-3849-7190
}

Resumen: En este ensayo el autor le da un vuelco a la tesis que sostuvo en su libro Didáctica y curriculum, Convergencia en los programas de estudio, publicado en el siglo pasado en donde plantea una complementariedad entre las propuestas del ámbito didáctico y curricular. Su aproximación actual desarrolla un análisis de la estructura epistémica de ambas disciplinas y cuestiona que en la misma subyace una idea de normatividad sobre lo que es deseable que acontezca en el aula y en los aprendizajes de los alumnos. Sostiene que en este caso lo que se busca es lograr una homogeneidad en los procesos de aprendizaje, pero al mismo tiempo muestra como esa estructura rompe con los aspectos normativos cuando aparece en escena el debate sobre el sujeto y su singularidad. La ruptura epistémica que se da en el surgimiento y evolución de ambas disciplinas, aunque permite sostener la búsqueda de lo homogeneo, al mismo tiempo defiende que sólo puede formarse un sujeto, a partir de sus procesos singulares. Para ello, plantea un análisis del conflicto ideológico en el que surgen ambas disciplinas, una en el siglo XVIl y otra en el XX, así como la forma como este conflicto es ignorado por los sistemas educativos que apuntan más a reconocer la parte normativa común a sus proyectos, que al reconocimiento de los rasgos específicos de los sujetos en formación.

Palabras clave: Didáctica. Formación. Curriculum. Curriculum integral. Sujeto.

Abstract: In this essay the author flips around the thesis he held in his book Didactics and Curriculum, Convergence in the Curriculum, published last century, where he proposes a complementarity between the proposals of the didactic and curricular field. His current approach is to develop an analysis of the epistemic structure of both disciplines and question that in it lies an idea of normativity about what is

Doctor en Pedagogía pela Faculdade de Filosofia e Letras da Universidade Autônoma do México; Doctor Honoris Causa por la Universidad de Buenos Aires. 
desirable to happen in the classroom and in the student learning. He argues that in this case what is sought is to achieve a homogeneity in the learning processes, but at the same time shows how that structure breaks with the normative aspects when the debate about the subject and its uniqueness appears on scene. The epistemic rupture that occurs in the emergence and evolution of both disciplines, although allows to sustain the search for the homogeneous, at the same time defends that the subject only can be formed based on its unique processes. To this end, he presents an analysis of the ideological conflict in which both disciplines arise, one in the 17th century and the other in the 20th, just as the way this conflict is ignored by education systems that aim more to recognize the common normative part to their projects, than to the recognition of the specific features of subjects in training. Keywords: Didactics. Training. Curriculum. Comprehensive curriculum. Subject.

Resumo: Neste ensaio, o autor produz uma virada na tese que sustentou em seu livro Didática e currículo: Convergência nos programas de estudos, publicado no século passado, no qual propõe uma complementaridade entre as propostas no campo didático e curricular. Sua abordagem atual é desenvolver uma análise da estrutura epistêmica de ambas as disciplinas e questionar se nela existe uma ideia de uma normatividade sobre o que é desejável que aconteça na sala de aula e na aprendizagem dos alunos. Ele sustenta que, nesse caso, o que se busca é alcançar uma homogeneidade nos processos de aprendizagem, mas ao mesmo tempo mostra como essa estrutura rompe com os aspectos normativos quando o debate sobre o sujeito e sua singularidade aparece em cena. A ruptura epistêmica que ocorre no surgimento e evolução de ambas as disciplinas, embora nos permita sustentar a busca pelo homogêneo, defende ao mesmo tempo que apenas um sujeito pode ser formado, a partir de seus processos singulares. Para isso, propõe uma análise do conflito ideológico no qual ambas as disciplinas surgem, uma no século XVIl e outra no século XX, bem como a maneira pela qual esse conflito é ignorado pelos sistemas educacionais que visam mais reconhecer a parte normativa comum do seus projetos, do que reconhecer as características específicas dos sujeitos em formação.

Palavras-chave: Didática. Currículo. Formação. Currículo integral. Sujeito.

Recebido em 22 de outubro de 2020

Aceito em 29 de dezembro de 2020

\section{INTRODUÇÃO}

Iniciamos este ensayo afirmando que su elaboración es una especie de retorno al origen, dado que una de mis primeras obras ${ }^{2}$ (DIAZ-BARRIGA, 1984, 2019). Texto escrito bajo el supuesto de que los programas de estudio que orientan el trabajo del aula son un espacio

2 Su primera edición fue de 1984, se hizo una edición corregida en 1996, misma que se ha reimpreso en 2019. 
de convergencia de ambas disciplinas. Pero este ensayo no es sólo un regreso a un tema de origen, sino al mismo tiempo corresponde a un análisis realizado desde una perspectiva diferente en el que se señalan algunos problemas de corte epistémico que tienen su origen en la intencionalidad formativa de estas disciplinas, así como en el desarrollo conceptual e institucional que cada una de ellas ha tenido en estos últimos cuarenta años.

Este trabajo se sustenta en dos tesis, la primera sostiene que toda propuesta curricular contiene de manera implícita una perspectiva didáctica; mientras que la segunda, si bien reconoce la imbricación de ambas especialidades, al mismo tiempo establece que las mismas operan en una permanente contradicción sobre el acto educativo, dado que parten de una concepción diferente de su proyecto educativo y de lo que se espera del mismo. El currículo es en algún sentido un tema del que se apropian los ministerios de educación o las instituciones educativas, quiénes tienen que velar por su cumplimiento; mientras que la didáctica es una disciplina pensada para el trabajo docente en el aula. El supervisor escolar que en su origen de colocaba del lado de la didáctica, hoy se ubica en el terreno curricular.

De aquí se desprende un conflicto implícito entre expertos curriculares y especialistas en didáctica. Lo que permite comprender que en algunos casos, como en la literatura estadounidense y la mexicana, la didáctica haya sido desplazada de la formación inicial de docentes, de las licenciaturas universitarias vinculadas con la educación y de los programas de posgrado en educación. En el sentir popular cualquier persona puede desempeñarse como docente. Mientras que el currículo responde a la organización de contenidos que permitirán la formación de ciudadanos productivos, con conocimientos que permitan una competencia internacional. Para lo cual se apoya en múltiples prescripciones de corte burocrático, la entrega de la planeación didáctica, el informe del avance del curso (entendido como avance en el tratamiento de los diversos temas que contiene el programa de estudios) y las evaluaciones a gran escala nacionales e internacionales, que finalmente determinan el grado de dominio de los contenidos por parte de los estudiantes. Dominio que sólo se puede mostrar de una forma determinada.

Los especialistas en educación que abordan el estudio de la disciplina curricular o didáctica, las analizan desde su propia estructura conceptual, en pocas ocasiones, perciben que algunos elementos de las mismas coinciden en el trabajo que se realiza en el aula.

El problema fundamental no es que no que no tomen conciencia de ese punto de encuentro o desencuentro, sino que la perspectiva que tienen del trabajo que corresponde realizar a los docentes y alumnos, se encuentra tensionada por lo que consideran que debe ser enseñado de acuerdo a los expertos curriculares, frente a los procesos de desarrollo personal y cognitivo que plantean los especialistas en didáctica. Unos buscan lograr la homogeneidad del resultado educativo, mientras que los otros atienden a las condiciones de desarrollo de cada ser humano. 
Más allá del esfuerzo que realizan los primeros para reconocer que hay una diferencia entre el curriculum formal y el real, finalmente siguen concibiendo que corresponde al docente apropiarse de ese curriculum establecido para desarrollarlo en el aula. Ciertamente que ya superamos la etapa en la que el curriculum pensado era concebido solamente como el conjunto de "instrucciones" que tienen los docentes para su actuar en el aula. En el plano discursivo los expertos curriculares reconocen la diferenciación que existe entre curriculum formal y curriculum real. Aunque cuando se formulan los proyectos de valoración del trabajo educativo $y$, en ocasiones del trabajo docente, las pruebas a gran escala se alinean a lo que establece el currículo. Con lo cual el currículo pensado adquiere preponderancia sobre la práctica curricular. La tarea docente es concebida de esta manera como la de quien se apropia del proyecto curricular para paulatinamente irlo aplicando en su aula.

Por el contrario quiénes se dedican al estudio de la didáctica, conceden mayor significación a los procesos de aprendizaje que se dan en el aula, a las múltiples interacciones que acontecen en una realidad educativa, donde alumnos y docentes realizan un encuentro, tanto para aprender como para formarse. $Y$ que en este mismo encuentro siempre circunstancial buscan identificar cuáles son las potencialidades del grupo de estudiantes con el que trabajan, pero también cuáles son las posibilidades del mismo. Identificar su ritmo y proceso de construcción o desarrollos de aprendizaje, preguntarse sobre que aspectos del contexto en que viven los alumnos se pueden convertir en situaciones problema, en interrogantes de aprendizaje o en enigmas (MEIRIEU, 2002) con la finalidad de proponer una secuencia didáctica que posibilite el aprendizaje. Ciertamente a los didactas les preocupa los procesos que los alumnos pueden desarrollar, antes de determinar solamente cuál es el contenido o los temas que tienen que ser abordados. En este sentido curriculum y didáctica se tocan en un punto: el aula, pero se tocan desde una perspectiva muy distinta con intencionalidades que no son compartidas.

De hecho identificamos que las tareas que institucionalmente se le piden al docente están más cercanas al campo curricular que al ámbito didáctico, mientras que las acciones de los docentes sólo se pueden construir desde la situación didáctica, haciendo en ocasiones un poco de lado al proyecto curricular. No resulta fácil, para los docentes lograr una convergencia de intencionalidades educativas tan diversas

\section{DIDÁCTICA SUS ORÍGENES Y ESTRUCTURA EPISTÉMICA}

Para comprender el significado pedagógico y social que tiene la didáctica es necesario atender a algunos aspectos que se pueden identificar en el origen de esta 
disciplina. En la lucha ideológica que acontece previa a la conformación de los sistemas educativos, pues mientras estos se fueron estableciendo en el siglo XIX con posterioridad a la conformación de los Estados Nacionales; el debate en la constitución de la didáctica como disciplina del docente se da dos siglos antes.

El surgimiento de la didáctica se encuentra en los antecedentes de lo que será la escuela moderna. Nace también en un profundo conflicto ideológico religioso de la reforma y contrareforma de fines del siglo XVI y el siglo XVIII, en una profunda lucha social por lograr una primera emancipación de la humanidad, brindar educación a todos nobles y campesinos, niños y niñas, es una forma de lucha política que acontece en ese marco religioso (HOWARD, 1976). En este período se generaron tres textos de relevancia significativa para entender el debate sobre el sentido de la formación en las dos perspectivas y en su evolución. Textos que se pueden considerar genealógicos en el establecimiento de los grandes temas didácticos.

En primer término podemos identificar el libro de la Compañía de Jesús publicado oficialmente en 1599, bajo la dirección de la Compañía de Jesús realizada por el padre Acquaviva, me refiero a la Ratio adque institutio studiorum societatis IESU (GIL, 1999), ${ }^{3}$ un texto inspirado en lo que posteriormente se ha denominado la pedagogía ignaciana (CHARM0T, 1952) que reconocen un conjunto de principios de la pedagogía que emana de la Ratio Studiorum, que han evolucionado con el cambio de época y el desarrollo de la psicología y la pedagogía. La Ratio Studiorum es el resultado de la experiencia que tuvo la compañía que inicialmente buscaba que se integraran a su congregación personas que ya poseían una formación humanista, filosófica y teológica.

Al no encontrar este perfil en los aspirantes, decide formar a jóvenes para esta tarea. Esto permite entender la razón por la que la Ratio se refiere a detectar el ingenio, talento intelectual, aptitud general, cualidad espiritual (entendida como la fidelidad a los preceptos religiosos) que habría que ubicar en los estudiantes. Frente a otros que denomina con capacidades medianas. De ahí la estructura del documento iniciar con reglas para el provincial, rector, profesor de sagrada escritura, de hebreo, de teología, filosofía y matemáticas. Y colocando en otro lugar lo que denomina y profesores de las clases inferiores: retórica, humanidades, gramática (suprema, mediana e ínfima). El método del trabajo en clase consiste según Gil en la prelección, esto es en la "claridad de la exposición en cualquier rama del saber, según la capacidad de los alumnos. A continuación la repetición múltiple de los

\footnotetext{
Encontrar la versión castellana del libro no fue tarea fácil, la ventaja que tiene el que edita Gil es que además de un buen estudio introductorio contiene el texto integro de La ratio Studiorum publicado en 1599 en versión castellana y su original en latín. La primera parte del libro está dedicada a presentar el sistema educativo de la compañía de Jesús, donde también explica como se fue construyendo la Ratio Studiorum y las versiones que tuvo. Esta introducción ocupa las primeras 54 páginas del libro. posteriormente tiene la versión integra del texto publicado en 1599. Es de destacar que en notas a pié de página el editor va señalando algunas modificaciones e inclusiones que se hicieron en el siglo XVIII y XIX al texto original La segunda parte del libro la dedica a desglosar, lo que denomina la pedagogía ignaciana, desde una perspectiva actual (GIL, 1999).
} 
alumnos [...] ly finalmente] las constituciones [repetir un tema en determinada hora ante todos los alumnos]." (GIL, 1999, p. 48). La propia Ratio establece la recomendación a los prefectos de "familiarizarse con el libro del Método de Estudio y que procure que sus reglas sean observadas por todos los alumnos" (GIL, 1999, p. 85), así como su obligación de “Oir de vez en cuando a los profesores, al menos una vez al mes y también de cuando en cuando leeer los apuntes tomados por los discípulos." (GIL, 1999, p. 88).

Plantea algunos tipos de exámenes: un examen para el ingreso donde además de la entrevista a cada aspirante se le pedirá que redacte un texto sobre temas humanísticos; un examen que podríamos considerar de avance del curso, que es corregido por "los rivales [del examinado] para que adviertan y corrijan publicamente, mencionando el precepto contra el que se faltó [...] mientras se hace esto los discípulos a solas consigo mismo lean y corrijan el original de sus composiciones" (GIL, 1999, p. 144) y, un tercer examen para poder acceder a estudios de filosofía y al cabo de estos otro para ser aceptado en los de teología.

Es importante hacer notar que el modelo de educación formulado por este documento no se refiere a la educación infantil, sino a la función de formar cuadros para ingresar a la compañía de Jesús o realizar determinada función en la sociedad. Se trata de un documento que antecede también a una dimensión institucional pues tanto la graduación de los conocimientos clasificados en menores y superiores, como las prácticas docentes y de los estudiantes, son reguladas por este texto, buscando que sean los rectores y los prefectos los responsables de cuidar que estos principios se lleven permanentemente a la práctica. El carácter prescriptivo de lo que posteriormente se constituirá en un modelo didáctico se encuentra asentado en este libro. De igual forma, es necesario precisar que algunos elementos pedagógicos de esta somera descripción se refieren a núcleos conceptuales que forman parte del debate didáctico actual.

Por su parte, Snyders (1974) plantea que el mérito de la Ratio Studiorum fue establecer un sistema de trabajo común en los distintos internados jesuitas con el fin de concentrar a los mejores alumnos en París; sin embargo hace un cuestionamiento pedagógico y social a su funcionamiento en el siglo XVII, planteando que era una institución fundamentalmente para nobles, donde se tenía que utilizar fundamentalmente el latín, ya que el uso de la lengua materna se consideraba como algo vulgar. El autor reconoce que la disciplina del internado tenía la función de formar la voluntad del sujeto para enfrentar las situaciones complicadas de su futura vida. ${ }^{4}$ 
Por su parte en la región central de Europa, el crecimiento y protección del movimiento protestante $y$, en particular, los representantes de la reforma radical que cuya cosmovisión era la igualdad de todos seres humanos: nobles y plebeyos, es el fermento donde se construiría la didáctica como disciplina para el trabajo docente. Aparece como otro punto significativo en el proceso de construcción de este campo pedagógico.

La publicación de Didáctica Magna, constituye la formalización de una disciplina pensada para el trabajo docente en el aula. La didáctica es concebida como la disciplina para que el docente pueda resolver el la tarea de enseñanza con sus alumnos, establece el modelo de enseñanza simultánea que se ha preservado hasta nuestros días. Esta obra escrita por un pastor protestante finca sus raíces tanto en el pensamiento clásico de los primeros autores romanos de occidente: Cicerón, Séneca, Homero entre otros, así como diversas referencias a autores cristianos (San Agustín) a a textos bíblicos. En particular Didáctica Magna muestra una inspiración en el pensamiento de la Reforma Radical (HOWARD, 1976), esto es la reforma que lucha contra la monarquía y se fundamenta en el pensamiento de Lutero. Situación que lleva a su autor, Comenio a cambiar su residencia permanentemente para huir de la inquisición.

Más allá de las referencias religiosas con las que la primera parte del texto está construido en esa sección el autor se dedica a justificar que el hombre en un ser para el aprendizaje, para desarrollar sus potencialidades

Esto lleva a Comenio a pensar que la educación debe de ser para todos niños y niñas, nobles y campesinos reunidos en el mismo espacio. Lo que deseo plantear es que Didáctica Magna tiene una perspectiva de cambio social muy significativa. Representa una lucha mucho más amplia que lo que suele asignarse a lo que se denomina escuela tradicional. Comenio señala en este sentido "deben admitirse en las escuelas a todos por igual, nobles y plebeyos, ricos y pobres niños y niñas." (COMENI0, 1982, p. 30) Sobre este tema profundiza "No existe ninguna razón por la que el sexo femenino deba ser excluido en absoluto de los estudios científicos." (COMENIO, 1982, p. 31).

Parte de una crítica muy dura a la forma como se trabajaba en las escuelas de maestros e incluso según los planteamientos de Snyders (1974) de las prácticas del internado jesuita de fines del siglo XVl, Comenio expresa: "Las escuelas han sido vulgarmente tenidas por terror de los muchachos y destrozo de los ingenios, la mayor parte de los discípulos tomando horror a las letras y los libros se han apresurado a acudir a los talleres de artesanos." (COMENI0, 1982, p. 38).

La forma corriente de educar [...] que tanto profesores como alumnos llegan a conocer la lengua latina a fuerza de un grandisímo trabajo, de inmenso fastidio, de infinito esfuerzo a costa de un largo tiempo [...] estoy 
completamente persuadido de que algún genio maligno, enemigo del género humano, ha introducido este método en las escuelas. (COMENIO, 1982, p. 39).

Estos planteamientos son la base de la construcción de su propuesta didáctica que reconoce la necesidad de trabajar en la escuela a partir de la lengua materna, recomendando como principio didáctico enseñar "una cosa a la vez", en "ir de lo sencillo a lo complejo", "de lo concreto a lo abstracto", temas que dominarán el método de enseñanza propuesto por el autor. Al mismo tiempo señala un modelo de trabajo escolar organizado por grados, en un trabajo simultáneo del docente ante grupo de estudiantes. Un maestro al frente, en un lugar donde pueda mirar a todos. En el fondo lo que está estableciendo es la estructura de un salón de clases de forma rectangular donde el maestro se encuentra frente a alumnos, quiénes que sentados en hilera de bancas, atiendan las enseñanzas del docente. Más tarde Aebli (1958) denominaría a este modelo como una didáctica sensualista, por el papel que da a los sentidos, a los objetos como parte del proceso de aprendizaje.

Un acierto/límite del planteamiento comeniano es que un único método resuelve los problemas de enseñanza. Si bien, resulta acertado el planteamiento de graduar el proceso de aprender a partir de temas simples, para avanzar hacia procesos más complejos. Sin embargo, la insistencia en un único método será la causa de que la evolución de la didáctica, sobre todo en algunos autores del siglo XX, se haya convertido en una cuestión normativa. Considerando al método como algo que debe seguir el docente $y$, no en un reto para organizar una secuencia de trabajo, acorde a las condiciones del grupo de estudiantes con los que trabaja.

A diferencia de la Ratio Studiorum, Comenio asigna al examen un lugar en el método de aprendizaje. Es la etapa última del método y su finalidad es "lograr que nada quede de error en los alumnos". El avance en la formación en su propuesta será resultado de su edad y no del resultado en un examen.

La última parte del libro se dedica a impulsar la organización de diversos tipos de escuela desde la materna hasta la academia, en esta parte la Comenio hace un esbozo de los contenidos que deben ser trabajados en cada etapa de formación.

Comenio como pastor protestante no sólo se preocupó por la formación religiosa del estudiante, sino que en el plano político buscó enseñar todo a todos, al mismo tiempo que estableció una forma de gradual enseñanza que hiciera de la escuela un espacio agradable de aprendizaje, empezando por trabajar en la lengua materna, siempre huyendo del brazo de la inquisición.

El triángulo de este debate epistémico en el que se construye la didáctica se cierra con Juan Bautista de La Salle, a quien se atribuye la elaboración de la Guia de las 
Escuelas Cristianas (LA SALLE, 1986) ${ }^{5}$ en el año 1720. Un texto que orientado a establecer una serie de normas sobre el funcionamiento de la escuela, concebido fundamentalmente para dar acceso a la educación a niños pobres, de ahí el tema de la gratuidad, promoviendo lo que Houssaye (2014) señala como el establecimiento de "una estricta organización y de normas precisas de funcionamiento cotidiano. Los alumnos asisten a las escuelas 40 horas a la semana durante 46 semanas durante el curso escolar." (HOUSSAYE, 2014, p. 247).

La Salle logra combinar ciertos elementos de la Ratio Studiorum, con algunos, quizá pocos de la propuesta comeniana, así como formular los propios. Se puede considerar La Guía de las Escuelas Cristianas como la reacción de la iglesia católica entre la propuesta didáctica que emanaba de la reforma protestante. Educar a pobres, enseñar a leer y a escribir en la lengua materna, reconocer grados de dificultad en lo que se tiene que aprender. Si bien se acepta que los alumnos aprendan latin, cuando ya dominan la lecto escritura.

A diferencia de Comenio, la Guía de las Escuelas Cristianas esta centrada en orden, vigilancia, silencio (del educador y de los educandos), y en la enseñanza de lectoescritura y operaciones básicas de aritmética. El tema del aprendizaje de las ciencias en su primera formulación no se encuentra. En cambio, el texto se encuentra constantemente con la enseñanza del catecismo, la oración y la reflexión.

Por otra parte de la Ratio Studiorum retoma el tema de los exámenes, realizados en forma permanente (una vez a la semana), exámenes que clasifican a los alumnos y los hacen moverse de lugar en el salón de clases, pasando a las bancas de adelante a los estudiantes que obtienen mejores resultados. Establece un sistema de notas que clasifica a los alumnos en 6 niveles: 1 los mejores, 2 los buenos, 3 los medianos, 4 los dudosos 5 repetidores y 6 los más repetidores (renvoayes en el original (HOYSSAYE, 2014, p. 265). Si bien es importante señalar que esta clasificación de los estudiantes no se traduce en una calificación

Su proyecto se desarrolla a partir de dos elementos un sistema jerárquico y oculto de vigilancia entre los alumnos, de suerte que todos sean responsables de señalar a los compañeros que alteran el comportamiento, vinculado a una propuesta de premios y castigos en el trabajo escolar. Es necesario reconocer que La Salle dosifica el empleo del castigo, evitando que se haga con expresiones emotivas de enojo o fastidio por parte de quien lo aplica.

Existen múltiples versiones del documento que orienta el trabajo de los Hermanos de las Escuelas Cristianas, congregación fundada por Juan Bautista de la Salle para dedicarse exclusivamente a la enseñanza. Al ser un libro podríamos decir institucional se hicieron varios ajustes al mismo conforme pasaron los años desde su aparición en 1720, hasta la versión del último capítulo en 1900. Para este trabajo tomamos la que presenta Saturnino Gallego (1986), por ser la original de su primera impresión y que se encuentra en su libro de la página 737 a 771 . 
Incorpora el principio de la repetición "Un método sintético que va de lo simple a lo complejo, que supone identificar las dificultades y enfrentarlas en las clases en forma sucesiva. Una clase de imitación y repetición son centrales." (HOYSSAYE, 2014, p. 246).

Según Houssaye será hasta el siglo XIX donde se impulse con mayor fuerza el tema de la homogeneización de los estudiantes, organizar la escuela una vez que en el estado nacional se empiezan a conformar los sistemas educativos. A través de esta organización buscar la "homogeneidad de capacidades, de edad donde el profesor hará cada vez más la clase como lección y después como curso [...] [la] pedagogía tradicional nace de una fuerte evolución." (HOUSSAYE, 2014, p. 263).

La estructura epistémica de la didáctica surgida en el debate religioso impregnará el signo de la tarea que se asigna al docente en su trabajo escolar. Formar al educando de acuerdo a un modelo preestablecido, lograr que adquiera un comportamiento (posteriormente ciudadano y laico) que le permita integrarse a la sociedad, educar a todos por igual.

La visión de la didáctica, como disciplina de la pedagogía va a evolucionar hacia fines del siglo XIX y principios del XX con el movimiento escuela activa, que buscará formular otro tipo de principios. Entre los cuáles se encuentran reconocer el interés y el trabajo del estudiante, así como los rasgos específicos de su edad. Mientras que ya en las últimas décadas del siglo XX al luchar por salirse del esquema normativo e instrumental en que fue concebida empezará a conformar distintas escuelas de pensamiento en su seno. Entre otros temas el abandono de una visión instrumental permitirá que asuma una reflexión más profunda sobre el proyecto político que subyace en la tarea de educación y en el trabajo en el aula, así como la dimensión histórica de su misma evolución. Lamentablemente este ensayo no alcanza a dar cuenta de todo ello, pero sin duda es una tarea pendiente en el trabajo educativo.

A finales del siglo XX y principios del XXI la didáctica experimentó un intenso trabajo por reestablecerse a sí misma como disciplina del campo de la pedagogía. Retomando la idea de que su objeto de estudio se encuentra centrado en los procesos de formación que acontecen en el aula, en este sentido se vinculó nuevamente a la concepción del trabajo docente y a considerar el alumno como el centro del trabajo educativo.

No alcanzamos en este momento a plantear todas las bases de las diferentes escuelas de pensamiento didáctico, unas centradas en la formación crítica y emancipación del ser humano en donde una figura relevante es el pensamiento de Freire en la múltiple obra que redactó; otras construidas desde la perspectiva de los procesos de desarrollo y formación que tienen los estudiantes, bien sea en la visión de construir enigmas, reconocer que el alumno construya el aprendizaje como un problema personal, que genere su deseo de aprender. 
Reconociendo que la voluntad del alumno no puede ser dominada desde afuera (MEIRIEU), mientras que otras perspectivas analizan los saberes docentes y la procedencia de esos saberes (curricular, disciplinario, pedagógico, experiencial) como es el caso de Tardif. Así como el desplazamiento que la didáctica ha sufrido a partir del movimiento de eficiencia escolar desarrollado a principios del siglo XX por los psicólogos experimentales de los EEUU, en donde de manera sútil pero significativa se reemplazó el tema de la formación por el de aprendizaje. Aprendizaje como nuevo foco del trabajo educativo, aprendizaje concebido como cambio conductual. Conductas redactadas como metas de aprendizaje que paulatinamente se fueron transformando la perspectiva de enseñar entorno a competencias, pensando de igual forma en la empleabilidad de los egresados. También se generaron acercamientos que provienen de la explosión de las tecnologías digitales y su afectación a la educación estableciendo nuevos temas como docencia distribuida (COLL), docencia centrada en el aprendizaje, así como la propuesta de construir aulas invertidas, donde el docente proporcione al estudiante una multiplicidad de opciones de acceso a la información, y permita que sean ellos los que generen procesos de apropiación de la misma. Con ello señalamos que como disciplina la didáctica se ocupa de lo que acontece en el ámbito del aula, luchando contra la visión que la restringe a una disciplina prescriptiva. Los grandes temas epistémicos de esta disciplina se encuentran en su surgimiento en el conflicto reforma-contrareforma. Conservando varios temas como objeto de trabajo y al mismo tiempo siendo parte de una lucha ideológica precisamente sobre como se concibe el proceso de formación del ser humano.

Ciertamente que la didáctica no ha permanecido en esta posición, en primer término se ha puesto en entredicho su carácter normativo, conservando la idea de ser una disciplina para el docente. En este aspecto la formación inicial de docentes en América Latina muestra un prisma de opciones, en algunos países caso Argentino la didáctica sigue siendo la piedra fundamental de la formación, en otros como en México sólo se trabaja una perspectiva sumamente reducida a la dimensión técnica de planificación escolar. En algunos programas de posgrado en educación definitivamente no existe y mientras el currículum ha adquirido una centralidad en el tema de la formación.

Como estableceremos, el currículum más allá de sus diversas escuelas de pensamiento que lo caracterizan, es percibido por los responsables de política educativa como el instrumento que contiene todo lo que deben aprender los alumnos en su formación, al docente le corresponde la tarea de atender que esos aprendizajes se adquieran mientras están a su cargo, mientras que el Estado o los Organismos Internacionales se encargan de elaborar las pruebas a gran escala para determinar el grado en que los estudiantes van manifestando estos logros educativos. En este punto se encuentra el pensamiento educativo dominante en este momento. Las pruebas a gran escala se han convertido en las grandes aliadas de los proyectos curriculares, reducidos a planes de estudios. El curriculum visto así responde más a los intereses de la institución que de los estudiantes y de los docentes. 
De ahí que varias escuelas didácticas contemporáneas pongan en entredicho esta visión y esta tarea instrumental que le asignan a la didáctica. Varias tendencias se pueden ubicar en este momento algunas francófonas, tales como los saberes docentes (TARDIF), el reto de que el estudiante construya su aprendizaje como proyecto personal (MEIRIEU) o el trabajo para una integración en el aprendizaje (ROEGIER), mientras que en el perspectiva Sajona sobresalen los planteamientos de Schumlan sobre los conocimientos de los docentes, o el estudio de Bain sobre lo que realizan los mejores docentes. Para América Latina el referente más relevante es Freire con toda su articulación entre formación del ser humano y política en pro de una educación emancipadora. El debate didáctico es por demás intenso en este momento.

Sin embargo, la perspectiva de lograr trabajar por proyectos y por problemas constituye el reto fundamental para transformar el trabajo educativo desde la perspectiva didáctica. Es en esta forma de trabajar donde se logra involucar de una manera más significativa a los estudiantes en la construcción de su proyecto de aprender. Temas que son resultado de los planteamientos realizados en el movimiento escuela activa que no logra trabajarse con el detalle que reclama en este ensayo.

\section{LA ESTRUCTURA EPISTÉMICA DEL CAMPO DEL CURRÍCULO}

El surgimiento del campo curricular como disciplina de las ciencias de la educación se da indudablemente en el siglo XX. Sin embargo, algunos historiadores refieren que el término currículo tiene antecedentes más remotos. Hamilton se hace la pregunta sobre la razón por la cuál en el siglo XVII los calvinistas de Glasgow adoptaran la palabra latina curriculum que significa en movimiento progresivo o una carrera para expresar su concepción educativa (HAMLTON, 1993, p. 198), la respuesta la encuentra en que en los registros de la Grammar School el término curriculum hacia referencia aun curso multianual que seguía cada estudiante (HAMLLTON, 1993, p. 199). De esta manera, según este autor, emergen en ese siglo dos temas tributarios en el origen de este término: orden y método. Sin embargo, en este momento no se podría hablar del curriculum como una disciplina establecida.

Su origen como campo de conocimiento se dará en la transición del siglo XIX al $X X$, siendo tres elementos los que influyeron en la genealogía de su desarrollo: En primer término la conformación de los incipientes sistemas educativos a cargo del estado nacional, como consecuencia de la revolución francesa; en segundo lugar, el tránsito de una sociedad agraria a una sociedad industrial, donde quedó más clara la necesidad de formar para una ciudadanía, de cara a las necesidades del empleo y potenció el pensamiento eficientista 
educativo que Hamilton reconoce que existía previamente en el Calvinismo; y, finalmente el desarrollo de teorías administrativas (la administración científica del trabajo) y de la psicología experimental a partir de las cuáles se empezaron a realizar los estudios sobre la eficacia escolar (DIAZ-BARRIGA, 2009).

El campo del currículo nace en un significativo ambiente por impulsar la eficiencia en el trabajo educativo y respondiendo a dos intereses intelectuales (HABERMAS, 1982). El dominante será la perspectiva curricular vinculada a la selección de contenidos en los planes de estudio bajo la lógica de preparar a los egresados para el mundo laboral (BOBBIT, 1918), lo que más tarda se reconocería como la versión técnica del currículo y, por otra parte, el planteamiento de Dewey (1902) en el que emplea el término currículo para referirse a los aprendizajes de algunas disciplinas por parte de los alumnos. En ese momento representando una de las corrientes de la escuela activa, lo que posteriormente permitiría reconocer la presencia de los sujetos de aprendizaje en los proyectos curriculares. El pragmatismo es el eje que permite anudar ambas perspectivas en su origen.

Mientras la didáctica es una disciplina concebida para el trabajo docente frente a los procesos de formación, el currículo se gesta frente a las necesidades de preparar a los trabajadores que reclama el proceso de industrialización, de ahí la impronta que lo vincula a las instituciones. Este tema es relevante dado que el currículo trazará el mapa de aprendizajes, como adquisiciones de desempeño de los estudiantes, que deben ser promovidas por los docentes si saben elegir los métodos adecuados y, cuyo círculo se cerrará, ante el papel que asignado a los exámenes a gran escala como instrumentos que permitirán medir y comparar los logros que muestren los estudiantes e indirectamente juzgar la labor de los docentes, de las instituciones escolares y de los sistemas educativos.

La aspiración de la visión técnica del currículo es formular que, en el marco del desarrollo industrial, la escuela necesita comprender cuáles son las exigencias y competencias que el mundo laboral requiere para traducirlas en proyectos de generación de conocimientos, habilidades, destrezas y actitudes en los estudiantes, en síntesis lo que en este momento se denomina competencias.

Por ello, cuando se mundializa hacia los años sesenta y setenta del siglo pasado, los sistemas educativos asumen la perspectiva curricular estrechamente vinculada a lo que se requiere para construir un plan de estudios, esto es la perspectiva técnica se impuso en la región, como un proyecto de dominación (CARNOY, 1993), aún cuando en esos años ya se había cuestionado en los EEUU ampliamente la perspectiva técnica del currículo, en particular a partir del significativo ensayo de Pinar (1989)sobre la reconstrucción el campo del curriculum. Así como los las aportaciones de otros autores como Jackson con la construcción del concepto currículo oculto o Schwab con su perspectiva del currículum moribundo o el currículo como práctica temas que no formarían parte de la primera etapa de difusión de este pensamiento 
en América Latina. La perspectiva técnica se impondría prácticamente en los años sesenta y setenta del siglo pasado en nuestros países (DÍAZ-BARRIGA; GARCÍA-GARDUÑO, 2014).

Dos aspiraciones acompañarán a la implantación de la visión técnica: la formación de un tipo de ciudadanía que permita al egresado de una institución escolar, desempeñarse eficazmente en una sociedad y, la asunción de que es factible una construcción científica de un plan de estudios. Tema, ese último, que ya Bobbit tenía muy claro al titular el capítulo seis de su libro "La construcción científica de un plan de estudios", planteando realizar lo que actualmente denominaríamos estudios del mercado ocupacional para determinar lo que necesita incluirse en un plan de estudios. Posteriormente en 1949 Tyler, enriquece esta visión bajo el concepto de Fuentes del Currículo, intentando conciliar la parte del mercado ocupacional, con la necesidad de incorporar las necesidades de los sujetos (los alumnos). "Ninguna fuente por sí sola puede considerarse válida para la selección de los objetivos de aprendizaje." (TYLER, 1949). Tema que finalmente adquiere una versión que tuvo más imparto cuando Taba (1962) formuló que la primera etapa de construcción de un plan de estudios nace de elaborar un "Diagnóstico de Necesidades".

Esta visión técnica que reduce lo curricular a los planes de estudios se convertirá en dominante en la región. Responde con mucha claridad a los intereses institucionales de tener criterios muy claros sobre lo que se debe enseñar en las diversas instituciones educativas. En este marco aconteció algo que no puede dejarse de mencionar. Si antes de la década de los años setenta los planes de estudio eran solamente un listado de materias que se encontraban impresos en una o dos hojas, o bien en el índice de un libro de texto para cada materia. A partir del ingreso de la propuesta curricular los planes de estudios son versiones de más de 100 páginas que contienen fundamentos (de muy diversa índole jurídicos, filosóficos, declaración de un posicionamiento pedagógico más declarativo que real), para traducir esto en perfiles de ingreso y egreso, objetivos en una primera etapa, actualmente competencias. En un caso desglosados en generales y específicos, aunque en ocasiones operativos, mientras que en el caso de competencias genéricas, específicas, hasta competencias de cada asignatura.

Un claro elemento guía todas estas propuestas técnicas, en ellas se parte el contenido hasta sus aspectos más puntuales, expresados en lo que cada detalle de la ejecusión que realiza un alumno (MAGER, 1990), estas partes dan opción a elaborar los objetivos conductuales o de aprendizaje como comportamientos observables que se pueden identificar en los alumnos, actualmente redactados en términos de comportamientos de competencias como logros de aprendizaje. El tema permanente es que al concluir una clase los estudiantes tienen que mostrar que a partir de ella pueden realizar una ejecusión que previamente no podían llevar a cabo. 
La responsabilidad profesional del docente se desprofesionalizó al concebirlo sólo como un planificador de actividades para impulsar estos comportamientos, como logros de procesos segmentados tanto en el área cognitiva, para ello se podría recurrir a la taxonomía de Bloom, como en lo que en ese momento se denominó área afectiva. Vale la pena señalar que actualmente en el marco de las competencias se denominan aprendizajes socio-emocionales, esto es, las competencias que permiten una buena preparación para la ciudadanía (respeto a los demás) como para la integración en el mundo laboral.

La perspectiva didáctica de la docencia se desvirtuó, al dejar de concebir su responsabilidad vinculada a la formación y al impulso del desarrollo de las capacidades y potencias que tiene el ser humano. La visión técnica redujo al docente a un programador eficaz de logros de aprendizaje. La aceptación mundial del papel de los exámenes a gran escala cerró el circuito de esta perspectiva, paradojalmente en el momento en que se insiste en profesionalizar la docencia, esta a su vez queda más circunscrita a un programador de actividades que permitan que los alumnos adquieran determinadas ejecusiones, las que están señaladas a través del currículo. ${ }^{6}$

Esta visión del campo del currículo estuvo acompañada de una estructura organizacional que ha sido difícil superar, que es la organización por asignaturas. Muchos cambios se han propuesto en los últimos cincuenta años para intentar darle vida, renovarla e incluso convertirla en factor de cambio. Hemos transitado del plan de estudios por asignaturas, a uno por objetivos de aprendizaje o conductuales. Cuando este modelo fue ampliamente cuestionado se avanzó hacia uno que buscó fundamentarse en el constructivismo, en el uso de tecnologías digitales, para posteriormente plantear la estructura curricular flexible y a fin/ comienzo del siglo plantear un proyecto organizado por competencias, pero en todos estos cambios la estructura por asignaturas pervive.

Un cambio fundamental se dió en todos estos años: aceptar que el trabajo fundamental de la tarea docente era lograr que el alumno muestre algo, en pocas ocasiones se llegó a la visión didáctica de que el alumno sólo aprende aquello que realiza, no aquello que escucha y aplica. Esta idea estuvo presente en el debate de principios del siglo XX en los EEUU cuando se empezaron a conformar lo que se llamarían "objetivos de aprendizaje", hoy renovados bajo el término competencias. Pero el lastre que estuvo presente en la organización de planes de estudio fue la segmentación de tiempos y contenidos a través de la organización por asignaturas.

Taba (1962) ha sido de las pocas autoras del campo del currículo que dedica un capítulo a estudiar la estructura de los planes de estudios, de la organización por asignaturas

6 Son en este sentido muy interesantes las argumentaciones de los defensores de la Prueba PISA, cuando plantean que en realidad sus contenidos están de alguna forma alineados a los currícula nacionales de enseñanza media. 
expresa: son planes que respetan la organización jerárquica del conocimiento, permiten establecer una secuencia gradual de los mismos (iniciando con los antecedentes), dan un lugar a cada asignatura, son fáciles de administrar. Para la autora revisar esta estructura remite a una especie de tarea arqueológica. No omite en señalar que el conocimiento se le presenta al alumno en la lógica de una disciplina y se le deja la tarea de ir integrando los saberes que proceden de diversas disciplinas como pueda, al mismo tiempo que señala que los contenido. De suerte que todos los intentos por mejorar estructuralmente la educación, finalmente se topan con una estructura curricular que tiene siglos.

En este sentido resulta por demás interesante atender los planteamientos de Beane (2010), autor que reconstruye como la historia desde principios del siglo XX aconteció una historia curricular que buscaba su integración. Aunque señala que los proyectos de integración curricular prácticamente quedaron olvidados en las propuestas de construir planes de estudio. En este punto vale la pena señalar que en el caso mexicano existen experiencias significativas a las que haremos mención un poco más adelante.

En esta reconstrucción histórica de la integración curricular es necesareio tener presente, que esta idea tiene su base en el pensamiento de John Dewey (1902) en donde se pregunta qué sentido tiene enseñarle al niño un poco de matemáticas, gramática o historia de manera segmentada. Si bien Beane, reconoce que en el debate estadounidense la integración curricular se olvidó y sólo volvió a emerger en la literatura y en los congresos del curriculum hasta finales de la década de los años ochenta del siglo pasado (BEANE, 2010, p. 16). El autor se refiere a experiencias en la educación básica donde plantea que los organizadores de la integración curricular "se extraen de la vida tal como se vive y experimenta" (BEANE, 2010, p. 17) que supone "aplicar conocimientoas a cuestiones e inquietudes que tienen importancia personal y social, [con lo cuall las fronteras entre las asignaturas separadas se difuminan" (BEANE, 2010, p. 17) y, finalmente demandan "una planificación participativa, conocimiento contextual [asil la integración curricular ofrece un amplio acceso al conocimiento a alumnos muy dispares." (BEANE, 2010, p. 17).

Más allá de las experiencias que en ese país se realizaron durante cerca de treinta años, en los años cuarenta los cuestionamientos al currículo integral en los EEUU los llevaron a ser marginados. Por ello se entiende que en la difusión del pensamiento curricular estadounidense los autores que reflejan este planteamiento hayan sido olvidados. Excepción significativa es Taba (1962) quien señala la existencia de una organización curricular denominada integral, considerando que uno de sus riesgos quedarse en grandes generalizaciones del conocimiento. Pero más allá de ella, prácticamente ningún autor de estos años hace referencia a ese tema. 
La tesis que nos ayuda a realizar estos planteamientos es reconocer que la estructura curricular, sea por asignaturas o integral, contribuye de manera significativa a orientar la práctica docentey el trabajo de los estudiantes.

En este sentido, reconocemos que han existido cuestionamientos a las asignaturas como las que realiza Goodson (2003) en su planteamiento de estudiar la historia de los contenidos que han formado parte de los planes de estudios, señalando que las asignaturas son parte de un microcosmos donde se esconden diversas intenciones político sociales del currículo. Considera que un estudio de esta historia permite develar el sentido que guarda esta caja negra en su sentido epistémico-político para la educación.

Los planteamientos de Goodson (2003) han tenido un impacto más en el sentido epistémico y político que en el plano de la acción curricular. No es que desvaloremos el papel que ello puede tener en el conocimiento de esta disciplina, sino que sencillamente para el interés que orienta este ensayo, no necesariamente se traducen en algo que modifique la práctica en los salones de clase.

La perspectiva del currículum que lo vincula a planes de estudio ciertamente es la dominante, aunque el campo no ha quedado reducido sólo a estos planes. Si bien no es objeto de este ensayo dar cuenta de todas las otras expresiones del campo quizá se puedan mencionar cinco como las más significativas, a reserva de excluir muchos planteamientos más y de sólo realizar una muy somera referencia a ellas en este momento.

En este sentido, no se puede desconocer la aportación que realizó Jackson (1992) con el concepto curriculo oculto, para dar cuenta de un conjunto de procesos y no intencionados que acontecen en la interacción escolar y del aula en particular. Tema que en su momento vino a convulsionar el debate en el campo.

Obligada es también una referencia al pensamiento de Schwab (1978), con sus tesis más conocidas de los años setenta del siglo pasado, el currículum moribundo y el currículo como una teoría práctica, aunque también es necesario reconocer aquellas perspectivas de este autor que han sido pasadas por alto, como su visión liberal del currículo en donde reconoce que la enseñanza no sólo debe difundir conocimiento, sino ayudar a impulsar la comprehensión del mismo. En esta ruta Schwab donde plantea un texto muy interesante sobre el papel del deseo ${ }^{7}$ en el desarrollo del conocimiento, así como el papel de lo estético sobre la intención lograda.

7 Este ensayo no alcanza a dar cuenta de todo lo que significa el papel del deseo en la educación. Término que aparece en Didactica Magna, si bien no con el significado que adquirirá a partir del psicoanálisis en el siglo XX y, que después es replanteado con significativa relevancia en el pensamiento didáctico de Meirieu y sorprendentemente en un capítulo de los trabajos recopilados en el libro de Schwab, al que estamos haciendo mención. Trabajar el deseo de aprender es y seguirá siendo un reto fundamental en la educación. 
Tampoco se puede negar la relevancia especial que William Pinar ha tenido en sus múltiples trabajos sobre la reconceptualización del currículo, los que inició en los años setentas con Curriculum Theorizing (PINAR, 1975), así como todo el trabajo que ha realizado para impulsar tanto la internacionalización del campo del currículo, como su visión cosmopolita que lo convierte, sin lugar a dudas, en uno de los impulsores más relevantes del campo en este momento.

Así como desde el impacto de la perspectiva marxista en la interpretación curricular desde los plantamientos de Michel Apple o de Henri Giroux entre otros.

Sin embargo, atendiendo al eje de nuestro ensayo deseamos focalizarnos en el tema integración curricular en virtud de que esta posición curricular, si bien vinculada a los planes de estudio, en su estructura epistémica impacta de una manera significativa el trabajo escolar, logrando modificar lo que se realiza en el aula. Constituye una posibilidad diferente de impulsar cambios significativos en el sistema educativo.

En el sistema de educación superior en México, de manera marginal, se impulsaron proyectos curriculares desde la perspectiva de la integración siendo los más significativos por el grado en el que lograron reinventar una estructura curricular fueron los siguientes: El sistema modular por objetos de transformación de la Universidad Autónoma Metropolitana. Unidad Xochimilco; el sistema modular del Centro Interdisciplinario de Ciencias de la Salud, Unidad Milpa Alta del Instituto Politécnico Nacional; y, el Plan de estudios experimental Medicina Integral A-36 de la Facultad de Medicina de la UNAM.

Sólo en el caso de la UAM-X, el sistema modular se aplicó a profesiones no sólo de las ciencias de la salud, incluyendo agronomía, sino también al ámbito de artes y diseño (arquitectura o diseño), así como a profesiones de las ciencias sociales (sociología, economía, psicología y comunicación) enfrentando otros retos de integración.

El reto de mantener en funcionamiento los 18 planes de estudio las ha llevado a buscar una permanente actualización de los mismos. Sin embargo, si se observa en la estructura de su sistema modular prevalece más el tiempo de trabajo fuera del aula, que lo que se suele denominar clases teóricas (UNIVERSIDAD AUTÓNOMA METROPOLITANA, 2020).

Por su parte el Plan de Estudios Experimental de Medicina Integral A-36, se pudo sostener hasta el año 1993 en donde fue cancelado por la misma Universidad (LAGUNA et al., 2020).

La dificultad de trabajar los contenidos profesionales a través de la resolución de problemas fue evidente, así como la forma como los docentes y estudiantes asumían permanentemente el reto de desarrollar estos conocimientos, al mismo tiempo que estudiaban y buscaban soluciones a problemas concretos de una comunidad, que requería de esos conocimientos profesionales. 
La experiencia de trabajo fue muy relevante y ha dado pie a la realización de múltiples investigaciones sobre ellas, de varias he dado cuenta más puntual (DIAZ-BARRIGA, 2020). Se puede resaltar cómo en el caso de los planes de estudio del Centro Interdisciplinario ha existido una evaluación periódica de los mismos, la que ha permitido reconocer sus aciertos, carencias y afrontarlas para ir subsanando las deficiencias identificadas. Se trata de varios planes de estudio de formación profesional (medicina, odontología, trabajo social, psicología) que aunque inicialmente sólo se desarrollan en una unidad del IPN, actualmente tienen vigencia en dos unidades institucionales (CENTRO INTERDISCIPLINARIO EN CIENCIAS DE LA SALUD, 2020).

Estos tres planes de estudio iniciaron su funcionamiento a fines de los años setenta y principios de los ochenta del siglo pasado. De los tres sólo los dos primeros funcionan en la actualidad. Los tres lograron subordinar los conocimientos de las asignaturas al establecimiento de un problema, el cual se convirtió en el ordenador o en la tarea a resolver a lo largo del módulo.

Esto significó modificar la disposición del aula escolar, buscando centrar el trabajo en lo que realiza el alumno y no en el docente, incluso desapareciendo físicamente un lugar asignado en el aula al docente, para incorporarlo como alguien que esta permanentemente interactuando con los avances de sus estudiantes. La clásica distribución del aula rectangular fue modificada, por un espacio más interactivo de alumnos y docentes; el espacio de aprendizaje se modificó de manera sustancial, pues los problemas elegidos que emanan de la realidad profesional tienen que ser estudiados no sólo en lo que dicen los libros, sino en lo que se encuentra en la realidad. Esto significó que docentes y alumnos distribuyeran su tiempo en ámbitos más allá de los circunscritos al aula o a las instalaciones universitarias, saliendo a estudiar los problemas en los lugares donde se encuentran, en ocasiones hospitales en el sector salud, pero también en las comunidades que asisten a ellos, en los lugares de siembra de los campesinos o en los de producción de carne de origen animal para consumo humano. Otro elemento en común en la elaboración de estos planes de estudios fue el apoyo que tuvieron en esos años de la Organización Panamericana de la Salud (OPS).

Si bien, se puede reconocer que en este momento son proyectos marginales con respecto al conjunto de la educación superior en México, ciertamente que su estudio e investigación permite observar como en ellos se desarrollan un conjunto de habilidades y estrategias para abordar el conocimiento diferente a la existente en los planes por asignaturas. Un cambio fundamental es no sólo que el estudiante se apropia del problema a resolver y de los conocimientos que reclama para ello, sino que al mismo tiempo asume una responsabilidad social de ofrecer alguna solución, a partir de contar con cierta inmersión en la comunidad donde desarrolla diversas estrategias para allegarse del conocimiento. Indudablemente la presencia del docente es fundamental, su tarea para nada es marginal, el docente no sólo 
acompaña el trabajo de los estudiantes, sino que permanentemente está orientado sobre la información a la que hay que acceder, aclarando dudas y confusiones, orientando el avance de los alumnos en la resolución del problema.

Al mismo tiempo es relevante reconocer que la literatura curricular con la que se elaboraron estos proyectos en los años setenta del siglo pasado, no se apoyó en ninguna referencia a los autores de la integración curricular, incluso ninguna a los autores clásicos del currículo, ni la noción de trabajo por problemas o por proyectos de la didáctica. Fue un intento muy significativo de avanzar hacia una ruta distinta de lo que denominaban educación tradicional, en ello radica parte de su originalidad, al mismo tiempo que partieron de un cuestionamiento a la formación didáctica y curricular que en ese momento ofrecían las universidades mexicanas.

La tarea docente en este perspectiva se vuelve un acto de mayor complejidad y reclama mayor involucramiento con todas las actividades de los alumnos. En algunos de estos casos, ante la carga que representa para un docente trabajar con los estudiantes diversos contenidos modulares vinculados a problemas específicos, guiarlos en la realización de la investigación del problema profesional seleccionado como eje estructurante de cada módulo, se ha avanzado hacia conformar equipos docentes para apoyar a un grupo específico de estudiantes.

Aquí radica la dificultad de gestión académica de este tipo de planes de estudios, una dificultad de gestión diferente a la que demanda la organización curricular por asignaturas, en donde los docentes son distribuidos por materias con una duración de 50 minutos por cada sesión de el trabajo con losestudiantes. De esta manera también podemosr econocer que la estructura curricular la que determina las condiciones del trabajo docente

\section{A MANERA DE EPÍLOGO}

Con la asunción de los proyectos educativos a nivel internacional centrados en eficacia escolar, que establecen mediciones que comparan resultados de los llamados logros de aprendizaje, que están asumiendo un modelo homogeneo de trabajo docente, estamos llegando al momento en que el énfasis del trabajo escolar está puesto en los resultados y no en los procesos formativos y cognitivos que subyacen en el aprendizaje.

En el caso de la integración curricular no se puede dejar de mencionar que en la actualidad es el sistema educativo finlandés el que busca avanzar hacia la integración curricular en la educación básica. En virtud de que los retos que reclama resolver tal integración las autoridades asumieron que su cambio curricular se proyectaría en un período 
de 15 años (del 2015 al 2030), con la finalidad de avanzar paulatinamente hacia esa meta. En este momento no es claro que lo logre, pero resulta a todas luces significativo que retome la línea de búsqueda de la tarea de integración (SILANDER, 2015).

\section{CONCLUSIONES}

Este ensayo se origina en la perspectiva de analizar la estructura epistémica de dos disciplinas la didáctica y el currículo, reconociendo parte de sus orígenes. Si bien busca dar cuenta de los rasgos fundamentales que marcaron la evolución de la disciplina, sólo establece grandes pinceladas sobre la evolución de ambos campos de conocimiento en el debate pedagógico contemporáneo. ${ }^{8}$ En el ensayo se ha buscado mostrar que tanto lo didáctico como lo curricular, al mismo tiempo que son deudores de los significados epistémicos que tuvieron en sus orígenes, esa estructura de alguna manera se preserva como una especie de "compulsión a la repetición", al mismo tiempo que existe una intensa lucha en sus desarrollos recientes por reencauzarse hacia otra perspectiva pedagógica o del campo de las ciencias de la educación. En este origen y en sus luchas posteriores se encuentra probablemente la fuente principal de su contradicción.

Currículo y didáctica de alguna forma convergen en un espacio: el aula, pero su convergencia no es complementaria unicamente como lo concebí al inicio de mis trabajos hace más de 35 años (DIAZ-BARRIGA, 1996), sino que en ella también se generan tensiones y conflictos dejando que cada docente en lo individual enfrente la forma como los atiende.

Es importante reconocer que el texto es profundamente deudor de múltiples vetas del debate contemporáneo en ambas disciplinas, vetas sobre las que será necesario profundizar.

Sin embargo, la tesis general del ensayo gira en relación a la necesidad de encontrar estrategias didácticas $\mathrm{y} / \mathrm{o}$ curriculares que permitan realizar un trabajo en el aula que permita apoyarse y profundizar en la tarea de formación del ser humano, en la construcción de un proyecto de aprendizaje por parte del alumno, así como lograr condiciones de un aprendizaje significativo. De ahí la importancia que concede a la necesidad de integración sea por el trabajo de proyectos y problemas desde lo didáctico o del currículo integral.

\footnotetext{
8 Teniendo como referencia del término contemporáneo la expresión que utiliza quien expresa Agamben (2006/2007, p. 2): "Contemporáneo es aquel que tiene la mirada fija en su tiempo para percibir no la luz sino la oscuridad."
} 


\section{REFERENCIAS}

AEBLI, H. Una didáctica fundada en la psicología de Jean Piaget. Buenos Aires: Kapelusz, 1958.

AGAMBEN, G. "¿2Que es lo contemporáneo". Texto inédito leído en la Facultad de Ares y Diseño de Venecia, 2006/2007. Disponible en: https://etsamdoctorado.files.wordpress.com/2012/12/agamben-que-es-lo-contemporaneo.pdf. Acceso en: 22 ago. 2020.

BEANE, J. La integración del currículo. El diseño de la educación democrática. Madrid: Morata, 2010.

BOBBIT, F. The curriculum. Houghton: Mifflin, 1918.

CARNOY, M. La educación como imperialismo cultural. México: Siglo XXI, 1993.

CENTRO INTERDISCIPLINARIO EN CIENCIAS DE LA SALUD. Unidad Milpa Alta IPN. Disponible en: https://www. cicsma.jpn.mx/. Acceso en: 22 ago. 2020.

CHARMOT, F. La pedagogía de los jesuítas. Sus principios y su actualidad. Madrid: Sapientia, 1952.

COMENIO, J. A. Didáctica magna. México: Edición Sepán Cuántos, Porrúa, 1982.

DEWEY, J. The child and the curriculum. Chicago: University of Chicago Press, 1902.

DIAZ-BARRIGA, A. De la integración curricular a las políticas de innovación en la educación superior mexicana. Revista Perfiles Educativos, México: Instituto de Investigaciones sobre la Universidad y la Educación, v. XLVV, n. 169, p. 160-179, 2020.

DIAZ-BARRIGA, A. Didáctica y curriculum - Convergencias en los programas de estudio. Ed. rev. México: Paidós Educador, 2019.

DIAZ-BARRIGA, A. Didáctica y curriculum - Convergencias en los programas de estudio. México: Nuevomar, 1984.

DÍAZ-BARRIGA, A. El docente y los programas de estudio. Lo institucional y lo didáctico. México: IISUE-UNAM, 2009.

DIAZ-BARRIGA, A.; GARCÍA-GARDUÑO, J. M. (coord.). Desarrollo del curriculum en América Latina. La experiencia de diez países. Buenos Aires: Universidad Autónoma de Tlaxcala-Miño y Dávila, 2014.

GALLEGOS, S. San Juan Bautista de la Salle. Madrid: Biblioteca de Autores Cristianos, 1986.

GIL, E. (org.). La pedagogía de los jesuítas, ayer y hoy. Madrid: Universidad Pontificia Comillas, 1999. 
GOODSON, I. Estudio del curriculum: casos y métodos. Buenos Aires: Amorrortu, 2003.

HABERMAS, J. Conocimiento e interés. Madrid: Taurus, 1982.

HAMILTON, D. "Orígenes de los términos educativos "clase" y "curriculum". Revista lberoamericana de Educación, Madrid: Organización de Estados lberoamericanos, n. 1, p. 187-205, 1993. Disponible en: https://rieoei.org/historico/oeivirt/rie01a06.htm. Acceso en: 22 ago. 2020.

HOUSSAYE, J. La Pédagogie traditionnelle. Una histoire de la pédagogie. Paris: Editions Fabert, 2014.

HOWARD, J. (org.). Textos escogidos de la reforma radical. Buenos Aires: Asociación Editorial La Aurora, 1976.

LA SALLE, J. B. Guía de las Escuelas Cristianas. In: GALLEGO, S. Obras completas de Juan Bautista de la Salle. Madrid: Biblioteca de Autores Cristianos, 1986.

JACKSON, P. La vida en las aulas. Madrid: Morata, 1992.

LAGUNA, J. et al. Plan de estudios experimental de medicina integral (Plan A-36). Disponible en: http:// hist.library.paho.org/Spanish/EMS/39524.pdf. Acceso en: 22 ago. 2020.

MAGER, R. Análisis de metas. México: Trillas, 1990.

MEIRIEU, P. Enseñar sí pero como. Barcelona: Octaedro, 2002.

PINAR, W. Curriculum theorizing: the reconceptualists. Berkley: McCutchan, 1975.

PINAR, W. La reconceptualización de los estudios del currículum. In: GIMENO, J. A. P. La enseñanza: su teoría y su práctica. Madrid: Akal, 1989. p. 231-240

SCHWAB, J. Science, curriculum and liberal education. Selected Essays. Chicago: The University Chicago Press, 1978.

SILANDER, P. Phenomenon Based Learning, 2015. Disponivel em http://www.phenomenaleducation.info/ phenomenon-based-learning.html. Acceso en: 22 ago. 2020.

SNYDERS, G. La pedagogía en Francia en los siglos XVII y XVIII. In: DEBESSE, M. G. Mialaret historia de la pedagogía II. Barcelona: Oikos Tau, 1974.

TABA, H. Curriculum development; theory and practice. New York: Harcourt, Brace \& World, 1962.

TYLER, R. Basic Principles of Curriculum and Instruction. Chicago: University of Chicago Press, 1949. 
UNIVERSIDAD AUTÓNOMA METROPOLITANA. Unidad Xochimilco. Disponible en: https://www.uam.mx/licenciaturas/licenciaturas_por_unidad.htm|\#\#oc. Acceso en: 22 ago. 2020.

Endereço para correspondencia: Universidad Nacional Autónoma de México, Centro de Estudios sobre la Universidad, Edificio de la Unidad Bibliográfica, Centro Cultural Universitario, Coyoacán, 04510, México, D.F., México; adbc49@gmail.com 